\title{
Genetic susceptibility to psoriasis and psoriatic arthritis: implications for therapy
}

\author{
H.L. Hébert, F.R. Ali, ${ }^{\star}$ J. Bowes, C.E.M. Griffiths, ${ }^{\star}$ A. Barton and R.B. Warren* \\ Arthritis Research UK Epidemiology Unit, University of Manchester, Manchester Academic Health Science Centre, Manchester, Stopford Building, Manchester, \\ M13 9PT, U.K. \\ *Dermatological Sciences, Salford Royal Hospital, University of Manchester, Manchester Academic Health Science Centre, Manchester M6 8HD, U.K.
}

\section{Summary}

\section{Correspondence \\ Richard Warren. \\ E-mail: richard.warren@manchester.ac.uk}

\section{Accepted for publication}

24 October 2011

\section{Funding sources}

H.L.H. is funded by a PhD studentship from Abbott; J.B. and A.B. are funded by Arthritis

Research U.K. (grant ref. 17552).

\section{Conflicts of interest}

R.B.W. has acted as a consultant and/or speaker for Abbott, Janssen Cilag, Leo Pharma, Pfizer and Schering Plough (now MSD). C.E.M.G. has acted as consultant for and/or received research grants from Abbott, Biogen-IDEC, Biotest, Centocor, Incyte, Janssen-Cilag, Leo Pharma, MSD and Pfizer. Other authors, none declared.
The era of genome-wide association studies has revolutionized the search for genetic susceptibility loci in complex genetic conditions such as psoriasis. There are currently 16 loci with confirmed evidence for association with psoriasis susceptibility but there is the potential for more to be discovered as the genetic heritability of the disease has not yet been fully explained. Many of the associated loci overlap with those for psoriatic arthritis. In contrast to psoriasis susceptibility, few studies have been performed to identify predictors of drug response in psoriasis. As large-scale collaborations and registries for psoriasis and psoriatic arthritis are established, it is likely that a genome-wide approach may be used as a more effective method of searching for genetic predictors of treatment response. However, candidate gene studies will still have a role; for example, it is likely that some disease susceptibility genes will also be markers of treatment response, based on evidence from other diseases. This review summarizes recent advances in investigating the role genetics plays in psoriasis susceptibility and contrasts these to advances made in psoriatic arthritis. Further, it describes the genetics of treatment response in the two diseases and indicates how susceptibility loci could be used to identify drug response in the future.

DOI $10.1111 / j .1365-2133.2011 .10712 . x$

Psoriasis is a complex genetic disease whose inheritance is not explained by simple Mendelian genetics, but rather arises due to the interaction of multiple genetic and environmental factors. ${ }^{1}$ Recent advances in genotyping technology have facilitated genome-wide association studies (GWAS), which in a short period of time have identified many genetic markers associated with psoriasis and highlighted novel mechanistic pathways. A greater understanding of the pathophysiological pathways causing psoriasis may provide future therapeutic targets and be of prognostic value in determining which treatment will be most effective in subsets of patients.

Psoriatic arthritis (PsA) is a condition that affects approximately $30 \%$ of people with psoriasis. ${ }^{2}$ Such patients have the clinical characteristics of psoriasis in addition to arthritis, softtissue swelling and enthesitis and, as with psoriasis, family studies have suggested a strong genetic component to the disease. $^{3,4}$

In this review, we discuss the known genetic associations with psoriasis susceptibility, including findings from recent GWAS, and compare genetic risk factors in psoriasis with those of PsA. We also summarize the results of studies under- taken to identify genetic predictors of drug response in both psoriasis and PsA.

\section{Genetics of psoriasis}

\section{Psoriasis susceptibility region 1}

The first genetic association studies in psoriasis were carried out in 1972 and were focused on the major histocompatibility complex (MHC) region on chromosome 6. ${ }^{5,6}$ Subsequently, human leukocyte antigen $\mathrm{C}$ (HLA-C) has been consistently shown to be associated with psoriasis. ${ }^{7-18}$ The 80-200-kb susceptibility region contained therein, designated psoriasis susceptibility region 1 (PSORS1), was identified through linkage studies. ${ }^{19-21}$ Two genes have been proposed as candidates for PSORS1. A C $>\mathrm{T}$ polymorphism at position +1243 in corneodesmosin (CDSN) was reported to be associated with psoriasis in $1999 .{ }^{22}$ In the same year, PSORS1 was narrowed to a region $89-200 \mathrm{~kb}$ telomeric of HLA-C with coiled-coil $\boldsymbol{\alpha}$-helical rod protein 1 (CCHCR1) as another possible candidate. ${ }^{21}$ Recent evidence suggests that HLA-C is the 
most likely causative gene for psoriasis. $^{23}$ However, this should be interpreted with caution as the use of selected single nucleotide polymorphisms (SNPs) in association studies and the presence of linkage disequilibrium between markers have made the identification of the causative mutation in PSORS1 difficult.

Subgroups of psoriasis are genetically heterogeneous for PSORS1. While early-onset psoriasis (onset before age 40 years) and guttate psoriasis ${ }^{24}$ are strongly associated with PSORS1, late-onset psoriasis (onset after age 40 years) appears not to be associated. ${ }^{25}$

\section{Genome-wide association studies}

Recent advances allowing rapid genotyping of hundreds of thousands of SNPs distributed across the genome have led to the advent of GWAS. ${ }^{26,27}$ Such GWAS do not rely upon prior hypotheses as to underlying molecular pathways and have been able to identify novel candidate genes and mechanisms associated with psoriasis. The GWAS performed thus far have all confirmed the association of PSORS1 with psoriasis but have also identified novel genetic loci containing candidate genes involved in the adaptive and innate immune responses and barrier integrity. Table 1 summarizes the genetic loci associated with psoriasis identified to date.

A number of important lessons can be drawn from these studies with regard to the pathways that contribute to disease (Fig. 1). Firstly, antigen presentation to the adaptive immune system by the innate immune system appears of paramount importance in triggering disease. This is supported by the fact that HLA-Cw6 remains the largest genetic susceptibility factor for psoriasis. Furthermore, the more recently identified endoplasmic reticulum aminopeptidase 1 (ERAP1) gene has been shown to interact with HLA-C, and is thought to regulate the antigenic peptides that bind to HLA-C. ${ }^{18,28}$

Secondly, the candidate genes identified from GWAS provide clues to the key inflammatory pathways underlying psoriasis. For example, the psoriasis-associated genes tumour necrosis factor alpha-induced protein 3 (TNFAIP3), TNFAIP3interacting protein 1 (TNIP1), v-rel reticuloendotheliosis viral oncogene homolog (REL), tyrosine kinase 2 (TYK2), TRAF3interacting protein 2 (TRAF3IP2) and nuclear factor of kappa light polypeptide gene enhancer in B-cells inhibitor-alpha (NFKBIA) are all regulators of the nuclear factor (NF)- $\kappa \mathrm{B}$ pathway. ${ }^{14,16,18,29} \mathrm{NF}-\kappa \mathrm{B}$ is a transcription factor involved in the regulation of the innate immune system and apoptosis. The TNFAIP3 protein product, A20, inhibits NF- $\kappa$ B and also interacts with TNIP1, thereby inducing keratinocyte hyperproliferation seen in psoriasis. Another key pathway involves the interleukin (IL)-23 signalling pathway, which regulates Th17cell activation. Variants within the genes, IL12B, IL23R and IL23A are all strongly associated with psoriasis susceptibility along with ZNF313/RNF114, which is also involved in T-cell activation. ${ }^{14,17,18,26,27,30}$ Activated Th17 cells produce IL-17, which in turn can activate the NFKB pathway via the ACT1 protein, which is encoded by another psoriasis susceptibility gene, TRAF3IP2. ${ }^{13,16}$

Thirdly, GWAS results have also provided clues to the mechanism by which the psoriasis phenotype is triggered in genetically susceptible individuals. For example, LCE3A, LCE3C and LCE3D in the late cornified envelope (LCE) gene cluster have been strongly associated with psoriasis and have important roles in skin barrier function. ${ }^{15}$ Candidate gene studies have also implicated the $\beta$-defensin gene cluster on chromosome $8 ; \beta$-defensins are antimicrobial peptides secreted in the epidermis to protect against microbial invasion. ${ }^{31}$ These clues from GWAS help to conceptualize how psoriasis may be triggered. Thus, impaired skin barrier function may allow an infectious pathogen or antigen to enter the body, be ingested and processed by antigen-presenting cells and presented to Th17 cells of the immune system. The association of both ERAP1 and HLA-Cw6 support the role of antigen presentation but whether this is an autoantigen or a foreign peptide, such as from a microbe, is unclear. The gene interferon induced with helicase C domain 1 (IFIH1), which has been associated with psoriasis in one GWAS, ${ }^{18}$ encodes a protein that mediates induction of interferon response to viral RNA, suggesting that at least one trigger may be viral. ${ }^{18}$ It is known that interferon$\alpha$ either induces or greatly exacerbates existing psoriasis when administered to patients being treated for conditions such as renal cancer ${ }^{32-35}$ and that it is upregulated in response to viral infection. ${ }^{36,37}$ In individuals genetically predisposed to psoriasis, the immune system is already primed and it is possible that activation by interferon may lead to development of the classical psoriasis phenotype.

Results of GWAS are also allowing some exploration of the genetic similarities and differences between psoriasis and PsA. While there have been fewer GWAS of PsA, it is clear from candidate gene studies that many psoriasis susceptibility loci are also associated with PsA. The allele HLA-Cw6 appears to be a common susceptibility gene for psoriasis and PsA in that it is found more commonly in both conditions than in healthy controls. Interestingly, however, it has a significantly higher frequency in psoriasis-only patients than those with PsA and has been associated with a less severe phenotype of PsA. ${ }^{12}$ The IL12B, IL23A, IL23R, LCE and TNIP1 gene variants are further examples of psoriasis susceptibility genes that have also been found to associate with PsA. ${ }^{12,38-40}$

Some genes appear to be associated only with PsA and not with psoriasis. Several candidate gene studies have reported that the HLA-B27 antigen is associated with PsA but not psoriasis. ${ }^{41,42}$ IL2 and IL21 were originally reported to be associated with PsA but not with psoriasis in a small GWAS by Liu et al.; ${ }^{12}$ however, a recent study has also reported association with psoriasis. ${ }^{43}$ Replication is required to establish if IL2 or IL21 confer susceptibility to either or both diseases. Association with the IL13 gene was originally reported in two psoriasis GWAS; ${ }^{14,18}$ subsequent analysis in one showed that the primary association was with PsA, and two further studies in which psoriasis and PsA samples were analysed also showed an association with PsA but not with psoriasis. ${ }^{4,45}$ Hence, 
476 Psoriasis and psoriatic arthritis: genetic and pharmacogenetic update, H.L. Hébert et al.

Table 1 Psoriasis (Ps) and psoriatic arthritis (PsA) associated genes

\begin{tabular}{|c|c|c|c|c|c|c|}
\hline Gene & $\begin{array}{l}\text { Chromosomal } \\
\text { region }\end{array}$ & Ps or PsA & Genetic marker & P-value & Odds ratio $(95 \% \mathrm{CI})$ & Reference \\
\hline \multirow[t]{2}{*}{ ERAP1 } & \multirow[t]{2}{*}{$5 q 15$} & \multirow[t]{2}{*}{ Ps } & rs151823 & $9.32 \times 10^{-9}$ & $0.89(0.85-0.92)$ & 28 \\
\hline & & & rs 27524 & $2.56 \times 10^{-11}$ & $1 \cdot 13(1 \cdot 05-1 \cdot 22)$ & 18 \\
\hline \multirow[t]{10}{*}{$\mathrm{HL} A-\mathrm{C}$} & \multirow[t]{10}{*}{$6 \mathrm{p} 21$} & \multirow[t]{3}{*}{ PsA } & rs13191343 & $2.32 \times 10^{-72}$ & $2 \cdot 37(2 \cdot 16-2 \cdot 61)$ & 16 \\
\hline & & & rs 10484554 & $6.86 \times 10^{-11}$ & $2 \cdot 4(1 \cdot 8-3 \cdot 1)$ & 12 \\
\hline & & & rs 2395029 & $1.86 \times 10^{-10}$ & $3 \cdot 2(2 \cdot 2-4 \cdot 6)$ & \\
\hline & & \multirow[t]{7}{*}{ Ps } & rs 10484555 & $1.81 \times 10^{-39}$ & $2 \cdot 8(2 \cdot 4-3 \cdot 3)$ & \\
\hline & & & rs2395030 & $2 \cdot 13 \times 10^{-26}$ & $4 \cdot 1(3 \cdot 1-5 \cdot 3)$ & \\
\hline & & & rs12191877 & $<10^{-100}$ & $2 \cdot 64^{\mathrm{a}}$ & 14 \\
\hline & & & rs 1265181 & $1.93 \times 10^{-208}$ & $22 \cdot 62^{\mathrm{a}}$ & 15 \\
\hline & & & rs 10484554 & $4.06 \times 10^{-214}$ & $4 \cdot 66(4 \cdot 23-5 \cdot 13)$ & 18 \\
\hline & & & rs 12191877 & $4 \cdot 21 \times 10^{-32}$ & $2.79(2.35-3.33)$ & 13 \\
\hline & & & rs3134792 & $1 \times 10^{-9}$ & $\mathrm{n} / \mathrm{a}^{\mathrm{b}}$ & 17 \\
\hline IFIH1 & $2 q 24$ & Ps & rs17716942 & $1.06 \times 10^{-13}$ & $1.29(1 \cdot 17-1 \cdot 43)$ & 18 \\
\hline \multirow[t]{4}{*}{ IL2/IL21 } & \multirow{4}{*}{$4 q 26-27$} & \multirow[t]{4}{*}{ PsA } & rs13151961 & $1.6 \times 10^{-3}$ & $0.64(0.49-0.84)$ & \multirow[t]{4}{*}{12} \\
\hline & & & rs7684187 & $1 \times 10^{-3}$ & $0.72(0.59-0.87)$ & \\
\hline & & & rs6822844 & $8 \times 10^{-3}$ & $0.72(0.56-0.92)$ & \\
\hline & & & rs6840978 & 0.013 & $0.77(0.62-0.95)$ & \\
\hline \multirow[t]{16}{*}{ IL12B } & \multirow[t]{16}{*}{$5 q 31-33$} & \multirow[t]{12}{*}{ Ps } & rs3212227 & $7.85 \times 10^{-10}$ & $0.64(0.56-0.73)$ & \multirow[t]{2}{*}{26} \\
\hline & & & rs6887695 & $4.08 \times 10^{-8}$ & $0.70(0.63-0.79)$ & \\
\hline & & & rs 10045431 & $1 \times 10^{-4}$ & $1.41(1 \cdot 21-1 \cdot 64)$ & \multirow[t]{3}{*}{27} \\
\hline & & & rs3212227 & 0.036 & $0.76(0.63-0.92)$ & \\
\hline & & & rs7709212 & $6 \times 10^{-3}$ & $0.76(0.65-0.89)$ & \\
\hline & & & rs2082412 & $2 \times 10^{-28}$ & $1 \cdot 44^{\mathrm{a}}$ & 14 \\
\hline & & & rs3213094 & $2.58 \times 10^{-26}$ & $0.78(0.74-0.81)$ & 15 \\
\hline & & & rs7709212 & $1.12 \times 10^{-20}$ & $0.80(0.77-0.84)$ & \\
\hline & & & rs2546890 & $1.29 \times 10^{-20}$ & $\mathrm{n} / \mathrm{a}^{\mathrm{b}}$ & 13 \\
\hline & & & rs953861 & $6.21 \times 10^{-18}$ & $\mathrm{n} / \mathrm{a}^{\mathrm{b}}$ & \\
\hline & & & rs3213094 & $4.93 \times 10^{-11}$ & $1.39(1.26-1.53)$ & 18 \\
\hline & & & rs6887695 & $5 \times 10^{-5}$ & $\mathrm{n} / \mathrm{a}^{\mathrm{b}}$ & 12 \\
\hline & & \multirow[t]{4}{*}{ PsA } & rs6887695 & $1.3 \times 10^{-3}$ & $0.69(0.56-0.85)$ & \\
\hline & & & rs 12188300 & $7 \cdot 33 \times 10^{-17}$ & $1.70(1.50-1.93)$ & 16 \\
\hline & & & rs3212227 & $1 \times 10^{-3}$ & $1.43(1.17-1 \cdot 76)$ & 39 \\
\hline & & & rs6887695 & $4.2 \times 10^{-5}$ & $1 \cdot 43(1 \cdot 18-1 \cdot 74)$ & \\
\hline IL13 & $5 q 31$ & Ps & rs20541 & $5 \times 10^{-15}$ & $1 \cdot 27^{\mathrm{a}}$ & 14 \\
\hline & & & & 0.0232 & $1 \cdot 12(1 \cdot 02-1 \cdot 24)$ & 18 \\
\hline & & PsA & rs1800925 & $6 \cdot 1 \times 10^{-5}$ & $1.33(1.16-1.53)$ & 45 \\
\hline & & & rs20541 & $8 \times 10^{-4}$ & $1 \cdot 27(1 \cdot 10-1 \cdot 45)$ & \\
\hline IL23A & $12 \mathrm{q} 13$ & Ps & rs2066808 & $1 \times 10^{-9}$ & $1 \cdot 34^{\mathrm{a}}$ & 14 \\
\hline & & PsA & rs2066808 & $9.05 \times 10^{-7}$ & $0.54(0.43-0.70)$ & 40 \\
\hline IL23R & $1 \mathrm{p} 31$ & Ps & rs7530511 & 0.014 & $0.78(0.66-0.91)$ & 26 \\
\hline & & & rs11209026 & $1.89 \times 10^{-4}$ & $0.63(0.50-0.79)$ & \\
\hline & & & p.Arg381Gln & $1.4 \times 10^{-4}$ & $0.49(0.35-0.68)$ & 27 \\
\hline & & & rs2201841 & $3 \times 10^{-8}$ & $1 \cdot 13^{\mathrm{a}}$ & 14 \\
\hline & & & rs11209026 & $7 \cdot 13 \times 10^{-7}$ & $1.49(1.27-1.74)$ & 18 \\
\hline & & & rs11209026 & $1.4 \times 10^{-4}$ & $0.56(0.41-0.76)$ & 12 \\
\hline & & PsA & rs11209026 & $8.3 \times 10^{-4}$ & $0.52(0.35-0.77)$ & \\
\hline & & & rs11209026 & 0.04 & $1.40(1.01-1.96)$ & 39 \\
\hline IL28RA & $1 \mathrm{p} 36$ & Ps & rs4649203 & $6.89 \times 10^{-8}$ & $1 \cdot 13(1.05-1.22)$ & 18 \\
\hline LCE & $1 \mathrm{q} 21$ & Ps & rs6701216 & $6.2 \times 10^{-5}$ & $1.45(1.21-1.75)$ & 12 \\
\hline & & & rs4112788 & $7 \cdot 13 \times 10^{-27}$ & $0.77(0.74-0.81)$ & 15 \\
\hline & & & rs4085613 & $6.69 \times 10^{-30}$ & $0.76(0.72-0.80)$ & \\
\hline & & & rs4845454 & $4.35 \times 10^{-29}$ & $0.76(0.72-0.80)$ & \\
\hline & & & rs1886734 & $2 \cdot 18 \times 10^{-28}$ & $0.76(0.73-0.80)$ & \\
\hline & & & rs4112788 & $3.32 \times 10^{-10}$ & $1.29(1 \cdot 19-1 \cdot 40)$ & 18 \\
\hline & & & LCE3B/C del & $3.8 \times 10^{-5}$ & $\mathrm{n} / \mathrm{a}^{\mathrm{b}}$ & 13 \\
\hline & & PsA & rs4085613 & $1.4 \times 10^{-3}$ & $1 \cdot 18(1 \cdot 07-1 \cdot 31)$ & 38 \\
\hline & & & rs4112788 & $1 \cdot 1 \times 10^{-3}$ & $1.19(1.07-1.32)$ & \\
\hline
\end{tabular}


Table 1 (Continued)

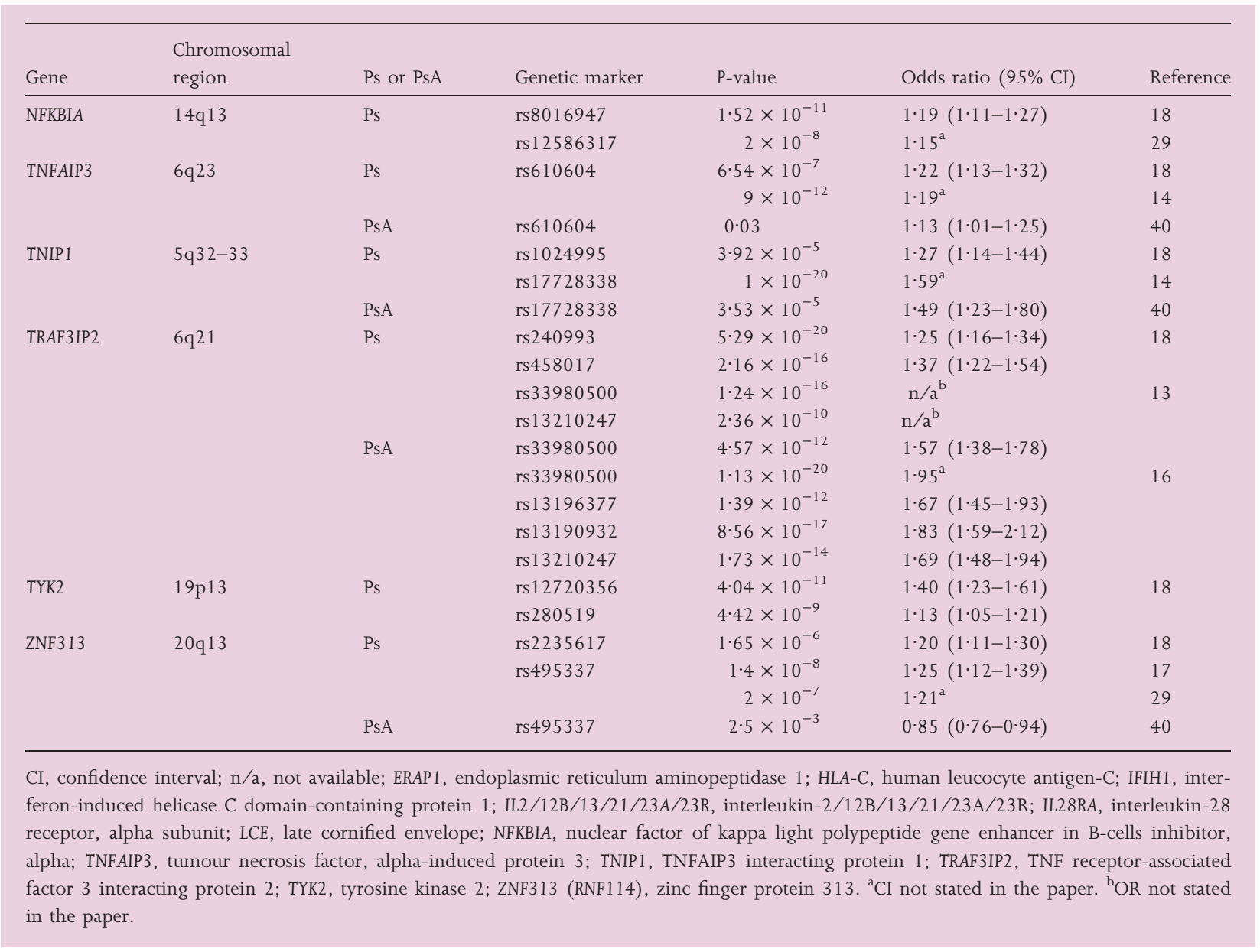

there are emerging examples of genes associating with PsA but not with psoriasis which support the concept that PsA is a 'disease within a disease'. It is likely that further genetic susceptibility loci will be identified for PsA which do not confer susceptibility to psoriasis.

Despite this progress, only about $25 \%$ of psoriasis heritability has been accounted for and this is due in part to the limitations of GWAS. Firstly, there is an inability to detect alleles which make a small contribution to disease phenotype. As P-value thresholds are raised to try to eliminate false positives, alleles with small effects are lost against the background of other tested markers. The only way to solve this problem is to increase statistical power by including more cases and more controls in the study. Secondly, GWAS is based on an assumption that a common disease will be caused by common variants. This neglects rarer variants that are present in the genome which may also contribute to psoriasis susceptibility. Thirdly, GWAS currently only include SNPs in the analysis. While SNPs are the most common form of variation in the human genome, other types of variation exist such as copy number variants and insertion/deletions which could also contribute to disease. Finally, GWAS cannot measure the effect of gene-gene interaction increasing or masking their contribu- tion to disease phenotype and epigenetic effects such as DNA methylation which influence gene expression. Indeed, the limited heritability identified to date for psoriasis is one reason why genetic findings about susceptibility cannot be immediately translated into clinical practice for population screening.

Nevertheless, there are a number of commercial companies offering genetic testing direct to the public. Many doctors do not know how to interpret the findings in the context of population risk. For example, for a disease with a lifetime prevalence of $1 \%$, a genetic risk factor that doubles the risk (to $2 \%$ ) still means there is a $98 \%$ chance of not developing disease. Secondly, the results are often based on case-control analyses, which may overestimate the risks associated with carriage of some genetic variants because they are based on hospital-based (more severe cohorts) rather than populationbased series of patients. Even for diseases in which genetic loci confer a high risk of disease, such as age-related macular degeneration, there is doubt as to whether screening of the general population for genetic markers would be helpful. ${ }^{46}$ In the case of psoriasis, the loci identified to date have improved our understanding of the aetiology of the condition but have not, as yet, led to tests with clinical utility. The area under the curve $(C)$ from a receiver operating curve analysis is one way 


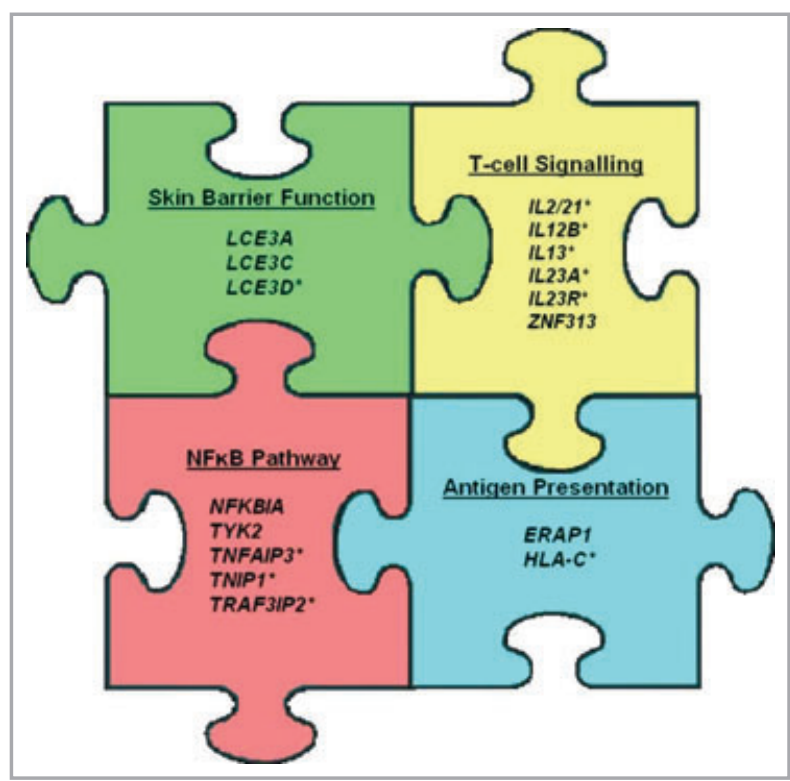

Fig 1. The psoriasis/psoriatic arthritis genetic jigsaw. Genetic variants within genes involved in four broad immunological processes have been found to be associated with psoriasis and psoriatic arthritis (PsA; marked with an asterisk). There are many more pieces required to complete the psoriasis/PSA genetic jigsaw but as large-scale collaborations allied with fine mapping studies are undertaken our understanding of these complex genetic disorders will evolve significantly. NFאB, nuclear factor $\kappa B$; ERAP1, endoplasmic reticulum aminopeptidase 1; HLA-C, human leucocyte antigen-C; IL2/12B/13/21/23A/23R, interleukin-2/12B/13/21/23A/23R; LCE, late cornified envelope; NFKBIA, nuclear factor of kappa light polypeptide gene enhancer in B-cells inhibitor, alpha; TNFAIP3, tumour necrosis factor, alpha-induced protein 3; TNIP1, TNFAIP3 interacting protein 1; TRAF3IP2, TNF receptor-associated factor 3 interacting protein 2; TYK2, tyrosine kinase 2; ZNF313, zinc finger protein 313 .

of assessing the clinical validity of a model. This plots the number of correctly predicted psoriasis cases (true positives) against the number of falsely predicted cases (false positives) as the number of 'watershed' risk alleles changes. A C-value of 0.5 shows no ability to predict disease, whereas a $C$-value of 1 shows perfect ability to predict disease. The $\mathrm{C}$-value should exceed 0.8 to be a good test. It has been estimated that, for effect sizes of $1 \cdot 25-1.50$ and assuming common allele frequencies, between 23 and 320 markers would be required to achieve $C>0.8$ based on genetic markers alone. However, if there are loci with large effects on response (higher odds ratios), the discriminatory ability is higher. Alternatively, a marker or group of markers may add to the ability of clinical factors to identify responders/nonresponders and be incorporated into a predictive model. ${ }^{47}$ It is likely that as our understanding of the genotype to phenotype correlation improves, predictions about the progression of psoriasis the condition may be possible.

Despite the fact that results from GWAS studies have not yet led to population-based screening programmes for psoriasis, GWAS studies can have clinical usefulness in identifying pathways which can in turn be the target of novel therapies. Indeed, the 'proof of concept' for this is the biological agent, ustekinumab, which is now licensed for use in psoriasis. Ustekinumab targets the IL-23 signalling pathway, which GWAS have highlighted as being crucial to the pathogenesis of psoriasis. Hence, GWAS may facilitate the development of novel therapies. However, it is unlikely that any new therapy will be effective for all patients with psoriasis. Which patients respond optimally to which therapies may be determined, in part, by their genetic profile; indeed this is one area where genetic research may translate into improved clinical care in the short to medium term.

\section{Pharmacogenetics of psoriasis}

\section{Translating predictors of disease to predictors of drug response}

Therapies used to treat psoriasis include topical, phototherapy and systemic and biologic agents. Selection of therapy is dependent on several factors including the severity of psoriasis, response to initial therapy and patient preference. Currently, psoriasis is incurable and therapies have nonselectively targeted inflammation and epidermal hyperproliferation. However, response to such therapies is variable. Pharmacogenetics is the study of whether and how genetic variation affects patient response to drugs, and includes identification of both efficacy and toxicity predictors. Ultimately, the aim of pharmacogenetic studies is to use knowledge of the DNA sequence to identify which drug will produce the best response with the least risk of side-effects for each patient. However, it is unlikely, firstly, that a single genetic test will reliably discriminate between responders and nonresponders, and, secondly, that it will be possible to identify individual gene profiles that will define who will respond well to treatment (personalized medicine) in the near future. Instead, identifying groups of patients likely to have good (or poor) responses to particular therapies, 'stratified medicine', may be more realistic and some studies have started to explore this.

For this review, only pharmacogenetic studies relating to systemic and biologic agents will be discussed. To date, all such studies in psoriasis and PsA have used a candidate gene approach where genes are selected for investigation based on knowledge of the biological pathways they lie on and the therapeutic agent to which response is being assessed. Studies using biologics have largely focused on the anti-TNF agents, while studies on systemic agents have focused on enzymes involved in drug transport and metabolism (Table 2).

\section{Systemic agents}

Methotrexate is the most researched systemic agent in the pharmacogenetics of psoriasis. ${ }^{48,49}$ It is often the first systemic 
Table 2 Genes containing polymorphisms associated with drug response in psoriasis and psoriatic arthritis

\begin{tabular}{|c|c|c|c|c|c|c|}
\hline Drug & Gene & $\begin{array}{l}\text { Chromosomal } \\
\text { region }\end{array}$ & Association & Genetic marker & P-value & Reference \\
\hline \multirow[t]{20}{*}{ Methotrexate } & \multirow[t]{2}{*}{ SLC19A1 } & \multirow[t]{2}{*}{$21 \mathrm{q} 22$} & Increased toxicity & rs 1051266 & 0.025 & \multirow[t]{5}{*}{53} \\
\hline & & & Drug discontinuation & rs 1051266 & 0.048 & \\
\hline & \multirow[t]{2}{*}{ TYMS } & \multirow[t]{2}{*}{$18 \mathrm{p} 11$} & Increased toxicity & 3’UTR 6 bp deletion & 0.025 & \\
\hline & & & No response & $5^{\prime}$ UTR 28 bp 3 repeat & $0 \cdot 048$ & \\
\hline & ATIC & $2 q 35$ & Drug discontinuation & rs2372536 & 0.038 & \\
\hline & \multirow[t]{9}{*}{$\mathrm{ABCC} 1$} & \multirow[t]{9}{*}{$16 \mathrm{p} 13$} & \multirow[t]{3}{*}{ Response } & rs35592 & $8 \times 10^{-3}$ & \multirow[t]{9}{*}{51} \\
\hline & & & & rs28364006 & 0.02 & \\
\hline & & & & rs2238476 & 0.02 & \\
\hline & & & \multirow[t]{6}{*}{ Adverse events } & rs2238476 & $0 \cdot 01$ & \\
\hline & & & & rs3784864 & 0.03 & \\
\hline & & & & rs 246240 & $6 \times 10^{-4}$ & \\
\hline & & & & rs3784862 & $2 \times 10^{-3}$ & \\
\hline & & & & rs 1967120 & 0.01 & \\
\hline & & & & rs11075291 & $8 \times 10^{-3}$ & \\
\hline & \multirow[t]{2}{*}{ ABCG2 } & \multirow[t]{2}{*}{$4 q 22$} & \multirow[t]{2}{*}{ Response } & rs13120400 & 0.03 & \\
\hline & & & & rs17731538 & $7 \times 10^{-3}$ & \\
\hline & ADORA2A & $22 q 11$ & Response & rs5760410 & 0.03 & \\
\hline & SLC19A1 & $21 \mathrm{q} 22$ & Response & rs 1051266 & 0.03 & \\
\hline & DHFR & $5 q 11$ & Response & rs1232027 & 0.02 & 56 \\
\hline & MTHFR & $1 \mathrm{p} 36$ & Increased toxicity & rs 1801133 & 0.04 & \\
\hline \multirow[t]{3}{*}{ Acitretin } & \multirow[t]{2}{*}{ VEGF } & \multirow[t]{2}{*}{$6 \mathrm{p} 12$} & No response & $\mathrm{rs} 833061 \mathrm{~T} / \mathrm{T}$ & 0.04 & \multirow[t]{2}{*}{59} \\
\hline & & & Response & rs833061 T/C & $0 \cdot 01$ & \\
\hline & TNF & $6 \mathrm{p} 21$ & Response & rs1799724 & 0.03 & 61 \\
\hline Adalimumab & TNF & $6 \mathrm{p} 21$ & Response & rs 1799964 & 0.04 & \\
\hline
\end{tabular}

therapy used to treat moderate to severe psoriasis but has variable interindividual responses, and approximately $30 \%$ of treated individuals develop side-effects, including hepatotoxicity and gastrointestinal toxicity. ${ }^{50}$ Methotrexate requires intracellular uptake and inhibits enzymes of the folate, purine and pyrimidine pathways, although the mechanism by which the drug exerts its effects in psoriasis and PsA are poorly understood. Nonetheless, the focus of many pharmacogenetic studies on methotrexate has been on the genes involved in its uptake and subsequent metabolism (Table 2). The largest of these studies reported a significant association between SNPs within the genes regulating the intracellular uptake of methotrexate, $A B C C 1$ and $A B C G 2$, and improved drug response. ${ }^{51}$ The $A B C$ genes are transporter genes of the ATP-binding cassette family. Five SNPs in ABCC1 were also found to be significantly associated with increased toxicity to methotrexate. In the same cohort, no associations with treatment response were found in genes coding for enzymes involved in methotrexate metabolism; folypolyglutamate synthase (FPGS), gamma-glutamyl hydrolase (GGH), methylenetetrahydrofolate reductase (MTHFR) and 5-aminoimidazole-4-carboxamide ribonucleotide transformylase (ATIC). ${ }^{52}$ Methotrexate toxicity has also been associated with a variant in the reduced folate carrier 1 gene (RFC1) gene, which is involved in regulating folate levels. ${ }^{53}$
Several studies have investigated genetic variation in the thymidylate synthase (TYMS) gene, which is involved in the production of thymidine triphosphate (TTP) required for DNA synthesis. Different variants have been investigated with conflicting results. ${ }^{53-55}$

The only pharmacogenetic study to be carried out exclusively in patients with PsA to date identified a polymorphism in the dihydrofolate reductase gene (DHFR) that was associated with better response to methotrexate. ${ }^{56}$ DHFR converts dihydrofolate to tetrahydrofolate, which is required for DNA synthesis and cell growth. Although this enzyme is inhibited by methotrexate it is not clear if this inhibition is crucial to its anti-inflammatory effects. The study identified another gene, MTHFR, which contained a SNP associated with increased hepatotoxicity. MTHFR is crucial for thymidine synthesis, necessary for cellular proliferation, and it is interesting to note the same SNP has been associated with a higher risk of developing adverse effects in patients with rheumatoid arthritis treated with methotrexate. ${ }^{57}$

Ciclosporin and acitretin are the two other major systemic drugs used to treat psoriasis. While no pharmacogenetic studies have yet been reported with ciclosporin, two have been performed with acitretin. The first of these examined polymorphisms in the apolipoprotein E gene (APOE) but found no 
association with response. ${ }^{58}$ The second reported that a variant at position -460 of the vascular endothelial growth factor (VEGF) gene was significantly associated with resistance to treatment with acitretin in psoriasis. ${ }^{59}$ These findings require replication for validity.

\section{Biologic agents}

Biologic agents are products that have been isolated from living organisms rather than synthesized by chemical methods. They are used to treat psoriasis and/or PsA in the setting of a contraindication and/or lack of efficacy of two or more systemic agents and phototherapy. Unlike systemic agents, they target specific biological pathways. Currently, there are five biologics used to treat psoriasis. Adalimumab (Humira ${ }^{\circledR}$; Abbott Laboratories Ltd, Maidenhead, U.K.), etanercept (Enbrel ${ }^{\circledR}$; Wyeth Pharmaceuticals, Maidenhead, U.K.) and infliximab (Remicade ${ }^{\circledR}$; Schering-Plough, Welwyn Garden City, U.K.) target the TNF- $\alpha$ pathway, important in the inflammatory process of several diseases including psoriasis. ${ }^{60}$ Ustekinumab (Stelara ${ }^{\circledR}$; Janssen-Cilag, High Wycombe, U.K.) targets IL-12 and IL-23, both of which have been implicated extensively in GWAS of psoriasis susceptibility, and alefacept (Amevive ${ }^{\circledR}$; Astellas Pharma Ltd, Wallisellen, Switzerland) targets T cells, but Switzerland is the only European country where it is licensed for use along with North America and Australia.

Two studies have reported genetic associations with response to anti-TNF therapy in psoriasis (Table 2). The first reported an association with a SNP in the TNF gene promoter region at position -1031 (rs1799964) $^{61}$ in a small sample cohort. The outcome measure used to assess response was based on patient-rated response and may have been subject to bias. $^{61}$ Hence, replication studies are required to confirm those findings. The second study combined patients with rheumatoid arthritis and ankylosing spondylitis and patients with PsA to analyse the effect of a TNF promoter region SNP at -308 (rs1800629) ${ }^{62}$ on response to three anti-TNF drugs. The study found significant association with increased response, but only 10 patients with PsA were included. Nonetheless, it is interesting to note that a meta-analysis of similar studies of this polymorphism in patients with rheumatoid arthritis treated with anti-TNF biologics has shown that it is significantly associated with response to etanercept and infliximab, respectively. ${ }^{63}$ Two other studies investigated the role of HLA-C, in determining response to biologics, but found no association. ${ }^{64,65}$

\section{Limitations and future work}

The pharmacogenetic studies undertaken to date have a number of limitations. Firstly, they often suffer from lack of power to detect association due to low sample size; typically studies have involved fewer than 100 patients. ${ }^{61,62}$ By contrast, GWAS of psoriasis susceptibility usually involve over 1000 patients and similarly sized control groups. Secondly, no independent replication is reported so the likelihood is that some of the associations will be false-positive findings. The ability to detect and replicate associations is hampered by the lack of agreed criteria for measuring drug response. For example, the Psoriasis Area and Severity Index (PASI) ${ }^{66}$ is often used to assess the clinical severity of psoriasis, but different percentage reductions in PASI, e.g. $90 \%, 75 \%$ and $50 \%$ have been used to define significant, clinically relevant improvement. Other limitations include the study of a single variant within a candidate gene with no attempt to investigate systematically an association with variants spanning the gene. Furthermore, factors such as alcohol intake and stress can be associated with nonresponse and studies often do not account for these possible confounders. ${ }^{67,68}$ Finally, the studies have largely selected candidate genes based on knowledge of the pathways involved in drug metabolism or signalling. However, a recent study in rheumatoid arthritis reported a disease susceptibility locus, protein tyrosine phosphatase, receptor type C (PTPRC), which appears to be predictive of patient response to TNF- $\alpha$ blockade. ${ }^{69}$ This has highlighted the possibility that disease susceptibility genes may also predict biologic drug response in psoriasis. Indeed, several psoriasis susceptibility genes encode proteins that lie on the pathways targeted by available therapies. For example, the IL12B, IL23R and IL23A genes are all involved in the IL-17-signalling pathway targeted by ustekinumab, while the TRAF3IP2 and TNFAIP3 gene products are involved in the TNF- $\boldsymbol{\alpha}$-signalling pathway which is a target of biologics including etanercept, adalimumab and infliximab. Pharmacogenetic studies in psoriasis, to date, however, have mainly investigated genes that have not been identified as conferring psoriasis susceptibility.

\section{Conclusion}

To date, 15 genes are associated with psoriasis, 11 genes are associated with PsA and 10 genes are associated with both, including HLA-C, IL12B, IL23R and TRAF3IP2. Variation within the IL13 gene locus and the HLA-B27 allele has been associated with PsA but not with psoriasis in most of the studies undertaken. While the debate as to whether all psoriasis susceptibility genes are also PsA genes is still in question, the fact that the HLA-Cw6 allele is significantly more associated with psoriasis compared with PsA suggests there may be some differences. Further study is needed to establish whether there is complete commonality of inflammatory pathways in both diseases. Future work in understanding which pathways are essential in which patient types may enable more therapies to be developed for these diseases.

It is clear that the pharmacogenetics of both psoriasis and PsA is very much in its infancy. Few studies have been undertaken in either disease and those that have are limited in both study design and size. The most researched drug thus far, methotrexate, appears to have plausible markers which may predict treatment response but all require validation. It is likely that one of the keys to stratified medicine in psoriasis will be identification of disease susceptibility genes which may also be predictors of treatment response. 


\section{What's already known about this topic?}

- Psoriasis and psoriatic arthritis are complex diseases with both genetic susceptibility and environmental triggers contributing to onset.

- Genome-wide association studies (GWAS) have advanced our knowledge of the genetics of both diseases.

- Psoriasis and psoriatic arthritis share a number of susceptibility loci.

- Patients show interindividual variation in their response to biologic and systemic drugs.

\section{What does this study add?}

- A summary of psoriasis susceptibility loci and pathways identified by GWAS and contrasted with those pertaining to psoriatic arthritis.

- Awareness that disease susceptibility alleles may also be predictors of drug response.

- Acknowledgment that pharmacogenetic studies in psoriasis and psoriatic arthritis are both few in number and weak, thereby identifying an area where further research is required.

\section{Acknowledgments}

F.R.A. is a National Institute for Health Research (NIHR) Academic Clinical Fellow; R.B.W. is a NIHR Clinical Senior Lecturer; C.E.M.G. is a NIHR Senior Investigator.

\section{References}

1 Brandrup F, Grunnet N, Henningsen $\mathrm{K}$ et al. Psoriasis in monozygotic twins: variations in expression in individuals with identical genetic constitution. Acta Derm Venereol 1982; 62:229-36.

2 Gladman DD, Antoni C, Mease P et al. Psoriatic arthritis: epidemiology, clinical features, course, and outcome. Ann Rheum Dis 2005; 64:ii14-17.

3 Moll JMH, Wright V. Familial occurrence of psoriatic arthritis. Ann Rheum Dis 1973; 32:181-201.

4 Myers A, Kay LJ, Lynch SA et al. Recurrence risk for psoriasis and psoriatic arthritis within sibships. Rheumatology 2005; 44:773-6.

5 White SH, Newcomer VD, Mickey MR et al. Disturbance of HL-A antigen frequency in psoriasis. N Engl J Med 1972; 287:740-3.

6 Russell TJ, Schultes LM, Kuban DJ. Histocompatibility (HL-A) antigens associated with psoriasis. N Engl J Med 1972; 287: 738-40.

7 Karvonen J. HL-A antigens in psoriasis with special reference to the clinical type, age of onset, exacerbations after respiratory infections and occurrence of arthritis. Ann Clin Res 1975; 7:301-11.

8 Henseler T, Christophers E. Psoriasis of early and late onset: characterization of two types of psoriasis vulgaris. J Am Acad Dermatol $1985 ; 13: 450-6$.
9 Murray C, Mann DL, Gerber LN et al. Histocompatibility alloantigens in psoriasis and psoriatic arthritis. Evidence for the influence of multiple genes in the major histocompatibility complex. J Clin Invest 1980; 66:670-5.

10 Brenner W, Gschnait F, Mayr WR. HLA B13, B17, B37 and Cw6 in psoriasis vulgaris: association with the age of onset. Arch Dermatol Res 1978; 262:337-9.

11 Tiilikainen A, Lassus A, Karvonen J et al. Psoriasis and HLA-Cw6. Br J Dermatol 1980; 102:179-84.

12 Liu Y, Helms C, Liao W et al. A genome-wide association study of psoriasis and psoriatic arthritis identifies new disease loci. PLoS Genet 2008; 4:e1000041.

13 Ellinghaus E, Ellinghaus D, Stuart PE et al. Genome-wide association study identifies a psoriasis susceptibility locus at TRAF3IP2. Nat Genet 2010; 42:991-5.

14 Nair RP, Duffin KC, Helms C et al. Genome-wide scan reveals association of psoriasis with IL-23 and NF-KB pathways. Nat Genet 2009; 41:199-204.

15 Zhang XJ, Huang W, Yang S et al. Psoriasis genome-wide association study identifies susceptibility variants within LCE gene cluster at 1q21. Nat Genet 2009; 41:205-10.

16 Huffmeier U, Uebe S, Ekici AB et al. Common variants at TRAF3IP2 are associated with susceptibility to psoriatic arthritis and psoriasis. Nat Genet 2010; 42:996-9.

17 Capon F, Bijlmakers MJ, Wolf $\mathrm{N}$ et al. Identification of ZNF313/RNF114 as a novel psoriasis susceptibility gene. Hum Mol Genet 2008; 17:1938-45.

18 Strange A, Capon F, Spencer CC et al. A genome-wide association study identifies new psoriasis susceptibility loci and an interaction between HLA-C and ERAP1. Nat Genet 2010; 42:985-90.

19 Nair RP, Henseler T, Jenisch S et al. Evidence for two psoriasis susceptibility loci (HLA and 17q) and two novel candidate regions (16q and 20p) by genome-wide scan. Hum Mol Genet 1997; 6:1349-56.

20 Trembath RC, Clough RL, Rosbotham JL et al. Identification of a major susceptibility locus on chromosome $6 \mathrm{p}$ and evidence for further disease loci revealed by a two stage genome-wide search in psoriasis. Hum Mol Genet 1997; 6:813-20.

21 Oka A, Tamiya G, Tomizawa M et al. Association analysis using refined microsatellite markers localizes a susceptibility locus for psoriasis vulgaris within a $111 \mathrm{~kb}$ segment telomeric to the HLA-C gene. Hum Mol Genet 1999; 8:2165-70.

22 Tazi Ahnini R, Camp NJ, Cork MJ et al. Novel genetic association between the corneodesmosin (MHC S) gene and susceptibility to psoriasis. Hum Mol Genet 1999; 8:1135-40.

23 Nair RP, Stuart PE, Nistor I et al. Sequence and haplotype analysis supports HLA-C as the psoriasis susceptibility 1 gene. Am J Hum Genet 2006; 78:827-51.

24 Asumalahti K, Ameen M, Suomela S et al. Genetic analysis of PSORS1 distinguishes guttate psoriasis and palmoplantar pustulosis. J Investig Dermatol 2003; 120:627-32.

25 Allen MH, Ameen H, Veal C et al. The major psoriasis susceptibility locus PSORS1 is not a risk factor for late-onset psoriasis. J Investig Dermatol 2005; 124:103-6.

26 Cargill M, Schrodi SJ, Chang M et al. A large-scale genetic association study confirms IL12B and leads to the identification of IL23R as psoriasis-risk genes. Am J Hum Genet 2007; 80:273-90.

27 Capon F, Di Meglio P, Szaub J et al. Sequence variants in the genes for the interleukin-23 receptor (IL23R) and its ligand (IL12B) confer protection against psoriasis. Hum Genet 2007; 122:201-6.

28 Sun LD, Cheng H, Wang ZX et al. Association analyses identify six new psoriasis susceptibility loci in the Chinese population. Nat Genet 2010; 42:1005-9. 
29 Stuart PE, Nair RP, Ellinghaus E et al. Genome-wide association analysis identifies three psoriasis susceptibility loci. Nat Genet 2010; 42:1000-4

30 Smith RL, Warren RB, Eyre S et al. Polymorphisms in the IL-12 $\beta$ and IL-23R genes are associated with psoriasis of early onset in a UK cohort. J Invest Dermatol 2008; 128:1325-7.

31 Hollox EJ, Huffmeier U, Zeeuwen PLJM et al. Psoriasis is associated with increased beta-defensin genomic copy number. Nat Genet 2008; 40:23-5

32 Funk J, Langeland T, Schrumpf E et al. Psoriasis induced by interferon-alpha. Br J Dermatol 1991; 125:463-5.

33 Harrison PV, Peat MJ. Effect of interferon on psoriasis. Lancet 1986; 2:457-8.

34 Neumann R, Pohl-Markl H, Aberer E. Parenteral interferon-alpha treatment of psoriasis. Dermatalogica 1987; 175:23-8.

35 Quesada JR, Gutterman JU. Psoriasis and alpha-interferon. Lancet $1986 ; 1: 1466-8$.

36 Siegal FP, Kadowaki N, Shodell M et al. The nature of the principal type 1 interferon-producing cells in human blood. Science 1999; 284: 1835-7.

37 Kadowaki N, Antonenko S, Lau JYN et al. Natural interferon alpha/beta-producing cells link innate and adaptive immunity. J Exp Med 2000; 192:219-25.

38 Bowes J, Flynn E, Ho $\mathrm{P}$ et al. Variants in linkage disequilibrium with the late cornified envelope gene cluster deletion are associated with susceptibility to psoriatic arthritis. Ann Rheum Dis 2010; 69:2199-203.

39 Filer $\mathrm{C}$, Ho P, Smith RL et al. Investigation of association of the IL12B and IL23R genes with psoriatic arthritis. Arthritis Rheum 2008; 58:3705-9

40 Bowes J, Orozco G, Flynn E et al. Confirmation of TNIP1 and IL23A as susceptibility loci for psoriatic arthritis. Ann Rheum Dis 2011; 70:1641-4.

41 Rahman P, Elder JT. Genetic epidemiology of psoriasis and psoriatic arthritis. Ann Rheum Dis 2005; 64:ii37-9.

42 Gladman DD, Farewell VT. The role of HLA antigens as indicators of disease progression in psoriatic arthritis. Multivariate relative risk model. Arthritis Rheum 1995; 38:845-50.

43 Warren RB, Smith RL, Flynn E et al. A systematic investigation of confirmed autoimmune loci in early-onset psoriasis reveals an association with IL2/IL21. Br J Dermatol 2011; 164:660-4.

44 Eder L, Chandran V, Pellett F et al. IL13 gene polymorphism is a marker for psoriatic arthritis among psoriasis patients. Ann Rheum Dis 2011; 70:1594-8.

45 Bowes J, Eyre S, Flynn E et al. Evidence to support IL-13 as a risk locus for psoriatic arthritis but not psoriasis vulgaris. Ann Rheum Dis 2011; 70:1016-19.

46 Jakobsdottir J, Gorin MB, Conley YP et al. Interpretation of genetic association studies: markers with replicated highly significant odds ratios may be poor classifiers. PLoS Genet 2009; 5:e1000337.

47 Kraft P, Wacholder S, Cornelis MC et al. Beyond odds ratios - communicating disease risk based on genetic profiles. Nat Rev Genet 2009; 10:264-9.

48 Ryan C, Menter A, Warren RB. The latest advances in pharmacogenetics and pharmacogenomics in the treatment of psoriasis. Mol Diagn Ther 2010; 14:81-93.

49 Warren RB, Griffiths CEM. The potential of pharmacogenetics in optimizing the use of methotrexate for psoriasis. Br J Dermatol 2005; 153:869-73.

50 Warren RB, Chalmers RJG, Griffiths CEM et al. Methotrexate for psoriasis in the era of biological therapy. Clin Exp Dermatol 2008; 33:551-4.
51 Warren RB, Smith RL, Campalani E et al. Genetic variation in efflux transporters influences outcome to methotrexate therapy in patients with psoriasis. J Invest Dermatol 2008; 128:1925-9.

52 Warren RB, Smith RL, Campalani E et al. Outcomes of methotrexate therapy for psoriasis and relationship to genetic polymorphisms. $\mathrm{Br}$ J Dermatol 2009; 160:438-41.

53 Campalani E, Arenas M, Marinaki AM et al. Polymorphisms in folate, pyrimidine, and purine metabolism are associated with efficacy and toxicity of methotrexate in psoriasis. J Invest Dermatol 2007; 127:1860-7.

54 Mandola MV, Stoehlmacher J, Muller-Weeks S et al. A novel single nucleotide polymorphism within the $5^{\prime}$ tandem repeat polymorphism of the thymidylate synthase gene abolishes USF-1 binding and alters transcriptional activity. Cancer Res 2003; 63:2898-904.

55 Gusella M, Bolzonella C, Crepaldi G et al. A novel G/C single-nucleotide polymorphism in the double 28 -bp repeat thymidylate synthase allele. Pharmacogenomics J 2006; 6:421-4.

56 Chandran V, Siannis F, Rahman P et al. Folate pathway enzyme gene polymorphisms and the efficacy and toxicity of methotrexate in psoriatic arthritis. J Rheumatol 2010; 37:1508-12.

57 Xiao H, Xu J, Zhou X et al. Associations between the genetic polymorphisms of MTHFR and outcomes of methotrexate treatment in rheumatoid arthritis. Clin Exp Rheumatol 2010; 28:728-33.

58 Campalani E, Allen MH, Fairhurst D et al. Apolipoprotein E gene polymorphisms are associated with psoriasis but do not determine disease response to acitretin. Br J Dermatol 2006; 154:345-52.

59 Young HS, Summers AM, Read IR et al. Interaction between genetic control of vascular endothelial growth factor production and retinoid responsiveness in psoriasis. J Invest Dermatol 2005; 126:453-9.

60 Griffiths CE, Barker JN. Pathogenesis and clinical features of psoriasis. Lancet 2007; 370:263-71.

61 Tejasvi T, Stuart P, Nair R et al. Preliminary pharmacogenetic assessment of tumour necrosis factor (TNF) polymorphisms in psoriasis: response to anti-TNF and systemic therapies. Br J Dermatol 2008; 159:1391 [Abstr.]

62 Seitz M, Wirthmüller U, Möller B, Villiger PM. The -308 tumour necrosis factor- $\boldsymbol{\alpha}$-gene polymorphism predicts therapeutic response to TNF $\boldsymbol{\alpha}$-blockers in rheumatoid arthritis and spondyloarthritis patients. Rheumatology (Oxford) 2007; 46:93-6.

63 Maxwell JR, Potter C, Hyrich KL et al. Association of the tumour necrosis factor-308 variant with differential response to anti-TNF agents in the treatment of rheumatoid arthritis. Hum Mol Genet 2008; 17:3532-8.

64 Costanzo A, Talamonti M, Botti E et al. HLA-Cw6 allele confers sensitivity to efalizumab treatment in psoriasis. Br J Dermatol 2008; 159:1392 [Abstr.]

65 Ryan C, Kelleher J, Collins P et al. A study to examine if the HLA$\mathrm{CW}^{*} 0602$ allele is a predictor of response to TNF- $\alpha$ inhibitors in the treatment of psoriasis. Br J Dermatol 2009; 161 (Suppl. 1):28 [Abstr.]

66 Fredriksson T, Pettersson U. Severe psoriasis - oral therapy with a new retinoid. Dermatologica 1978; 157:238-44.

67 Gupta MA, Schork NJ, Gupta AK et al. Alcohol intake and treatment responsiveness of psoriasis: a prospective study. J Am Acad Dermatol 1993; 28:730-2.

68 Heller MM, Lee ES, Koo JY. Stress as an influencing factor in psoriasis. Skin Therapy Lett $2011 ; 16: 1-4$.

69 Cui J, Saevarsdottir S, Thomson B et al. Rheumatoid arthritis risk allele PTPRC is also associated with response to anti-tumor necrosis factor alpha therapy. Arthritis Rheum 2010; 62:1849-61. 